\title{
ST Elevation and Syncope in A Young Patient with Covid-19 Disease: Brugada or Coronary Syndrome
}

\author{
Baris SENSOY*, Fatih LEVENT, Nur SENSOY and Elif GÜÇLÜ \\ Department of Cardiology Clinic, Health Sciences University Bursa Yuksek Ihtisas Training and Research Hospital, Turkey
}

*Corresponding author: Baris Sensoya, Department of Cardiology Clinic, Health Sciences University Bursa Yuksek Ihtisas Training and Research Hospital, Turkey.

Received Date: July 06, 2021

Published Date: July 22, 2021

\begin{abstract}
Coronavirus disease 2019 (COVID-19) has been reported to cause cardiovascular complications such as myocardial injury, thromboembolic events, arrhythmia, and heart failure. St segment elevation seen in electrocardiogram of COVID-19 patients may be associated with many diseases including acute myocardial infarction, myocarditis, Brugada syndrome and right ventricular pressure overload. Febrile states may unmask certain Brugada syndrome patients and precipitate ventricular arrhythmias. We present a case of a patient with coronavirus disease 2019 (COVID-19) and ST elevation arrived at our Emergency Department after syncope with loss of consciousness occurred during high fever.
\end{abstract}

\section{Introduction}

The COVID-19 pandemic is a major health crisis affecting several nations, with increasing cases and confirmed deaths reported to date [1]. The severe inflammatory response to COVID-19 results in a febrile illness in the vast majority of patients [2]. These patients present to the emergency department in different clinical scenarios and may require treatment modalities other than COVID-19 disease related specific and supportive treatment. Multiple potentially overlapping mechanisms, particularly inflammation, are responsible for underlying cardiovascular complications such as myocardial injury, thromboembolic events, arrhythmias, and heart failure. The reported cardiac injury may be a result of direct viral invasion of cardiomyocytes, increased metabolic demand, immune activation, or microvascular dysfunction. Cardiac arrhythmias have also been reported with a wide range of implicated contributory factors, ranging from direct viral myocardial injury, as well as other factors, including at-risk individuals with underlying inherited arrhythmia syndromes. The presenting cardiovascular symptoms include chest pain, dyspnoea, and palpitations [3]. Atypical complaints of patients are a problem that may cause complexity in terms of differential diagnosis. Although the patients are evaluated in detail for cardiac complications at the time of admission, the differential diagnosis of cardiac complications can be confusing.

\section{Case Presentation}

A 36-year-old man without relevant past medical history presented to the emergency department after syncope with loss of consciousness (40 seconds). He began to suffer from dyspnea, cough and fever 6 days before. His body temperature was $38.8{ }^{\circ} \mathrm{C}$ and was given antipyretic therapy $500 \mathrm{mg}$ of paracetamol 2 hours before applying. The syncope occurred in the bedroom shortly after the patient got out of bed, without any prodromal symptoms and with a very brief loss of consciousness. Despite being treated with amoxicillin for four days the shortness of breath had increased. The patient also complained of chest pain radiating to the back and after his unconscious period he was making senseless gestures for 2 hours. He did not report a history of syncope before, and there was no relevant family history. 


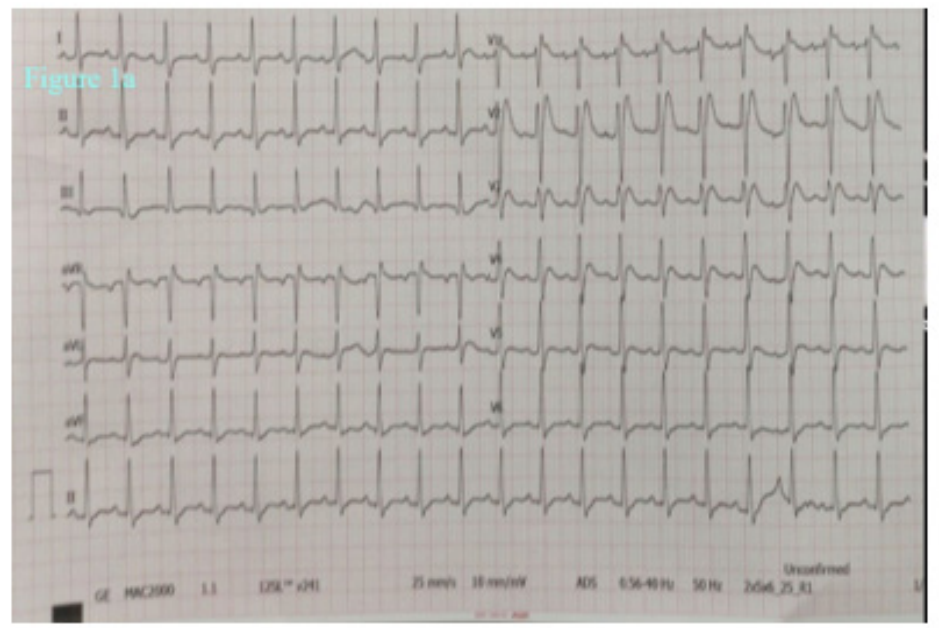

Figure 1a: The saddle-back ST-segment elevation morphologies most evident in derivation v1.

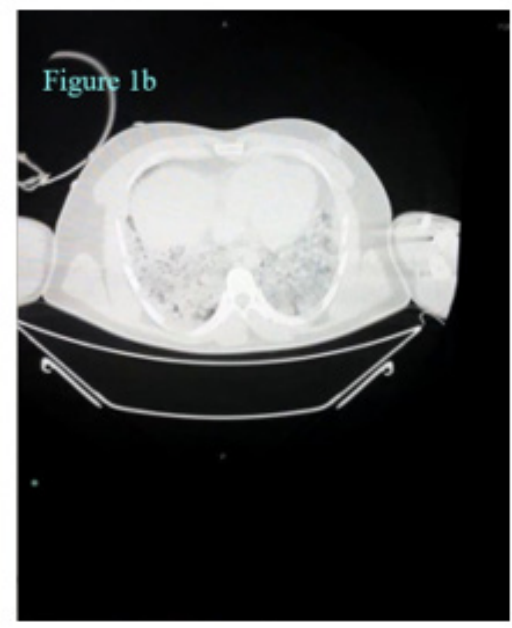

Figure 1b: Chest CT demonstrated consolidation that extensively covers both lung parenchymas.

In the emergency room the patient was sub febrile $\left(37.2^{\circ} \mathrm{C}\right)$ but noted to be tachycardic (117 beats/minute), hypotensive (80/50 $\mathrm{mmHg}$ ), hypoxemic (SpO2 79 \%) and had tachypnoea (respiratory rate 32 breaths per minute) with unaware and agitated behavior. On examination, he had increased work of breathing and diminished aeration in lung fields bilaterally. His neurological examination was normal. His initial work up revealed an elevated C-reactive protein (468 mg/l), lactate dehydrogenase (2090 U/L), kreatinin (1.5 mg/dl), AST (328 U/L) ALT (212 U/L), kreatin kinaz (1659 $\mathrm{U} / \mathrm{L})$, D-dimer $(8.8 \mu \mathrm{g} / \mathrm{ml})$, ferritin $(2484 \mathrm{ng} / \mathrm{ml})$ and troponin $\mathrm{T}$ levels (42 pg/ml). White blood cell count was $11900 \mathrm{mcl}$ and his electrolyte levels were normal. The 12-lead electrocardiogram (ECG) showed a sinus tachycardia of 121 beats per minute and ST elevation mimicking a Brugada type 3-like ECG pattern in lead V1 (Figure 1a). Considering a preliminary diagnosis of acute coronary syndrome with st-elevation, the patient was consulted to cardiology. Transthoracic echocardiogram was performed, which showed preserved cardiac function with dilated right chambers and an estimated systolic pulmonary artery pressure of $45 \mathrm{mmHg}$. Also, minimal $(<1 \mathrm{~cm})$ pericardial effusion was noted. The diagnosis of acute coronary syndrome was rejected. In order to rule out pulmonary embolism and detailed examination of lung parenchyma computed tomography (CT) of the chest was planned. Chest CT demonstrated bilateral extensive consolidations (Figure 1b). The patient's nasal/oropharyngeal swab was tested for SARS Cov-2 RT-PCR and it came out to be positive.

Due to his respiratory failure the patient was admitted to the intensive care unit of emergency room for continuous electrocardiographic monitoring and started treatment with high-flow oxygen inhalation. Although monitorization showed no significant arrhythmic events, including premature ventricular contractions and no sustained ventricular tachycardia, within 
3 hours of hospitalization, despite intubation and advanced respiratory support the patient rapidly decompensated, developing persistent hypoxemia and bradycardia, which ultimately necessitated a cardiopulmonary resuscitation with asystole. The patient died despite advanced life support.

\section{Discussion}

COVID-19 is a newly recognized infectious disease that has spread throughout the world, mainly manifested by fever, pneumonia, and respiratory failure. Recent studies reflect a wide range of cardiovascular complications and have associated this disease with an increased risk of arrhythmias and ECG abnormalities during hospitalization for COVID-19 pneumonia $[3,4]$. In the literature, there are patients who applied to the emergency department with ST elevation and COVID-19 disease, needed emergency coronary angiography or a subcutaneous implantable cardioverter-defibrillator $[5,6]$. Besides from direct viral invasion of cardiomyocytes, inflammation (potentially the role of IL-6), increased metabolic demand, immune activation, micro vascular dysfunction have been suggested as the underlying mechanisms for the thromboembolic events both in the venous and arterial systems related to the Covid-19 disease. Disease associated arrhythmia scenarios are tried to be explained by either direct viral myocardial damage and predisposed individuals with underlying inherited arrhythmia syndromes like Brugada syndrome [7-9].

Brugada syndrome is a sodium channelopathy characterized by the presence of ECG showing right bundle branch block and ST-segment elevation in the precordial leads. Brugada syndrome is diagnosed by the specific ECG pattern along with either family history of sudden cardiac death in a family member that is $<45$ years old or type 1 ECG in relatives, dysrhythmia-related symptoms (syncope, seizure, nocturnal agonal respiration), or ventricular tachycardia or ventricular fibrillation (VF), and patients are considered to have Brugada pattern if asymptomatic. Brugada syndrome is categorized into three types. Type 1 (coved type) involves ECG showing $\geq 2$-mm ST-segment elevation in one or more right precordial leads (V1 to V3) followed by an inverted T wave, occurring either spontaneously or after provocative drug test with sodium-channel blocker (flecainide, procainamide); type 2 (saddle-back type) has $\geq 0.5$-mm ST-segment elevation in one or more precordial leads followed by a saddle-back morphology to the ST elevation; and type 3 can show either coved or saddleback morphologies, but with $<2$-mm ST-segment elevation. Type 1 is diagnostic of Brugada syndrome, whereas Types 2 and 3 are suggestive [10].

Mediated by a cytokine-induced inflammatory cascade, COVID-19 incites high fevers, potentially instigating Brugada pattern changes. This change may be secondary to the temperature-dependent, pre-mature sodium channel inactivation found in SCN5A mutation, which is a major ionic abnormality responsible for the phenotype of Brugada pattern. Also feverinduced heart rate increase was suggested for the unmasking of the Brugada type 1 ECG pattern [11]. When Brugada syndrome is suspected, physicians should consult a cardiologist and consider ICD placement, depending on their risk of VF, though this risk stratification continues to be a controversy [10]. It was shown in a study including patients with Brugada syndrome that more than half of the study cohort had experienced syncope or cardiac arrest in the setting of a fever [12]. Different from this study, $\mathrm{Wu}$ and colleagues described the potential COVID-19-associated risks in known patients with Brugada syndrome. They suggested patients with Brugada syndrome to immediately attend the emergency department who develop high fever $\left(>38.5^{\circ} \mathrm{C}\right)$ despite paracetamol treatment if: (a) they had a sodium channel disease, (b) are under 26 years old or over 70 years, (c) had a spontaneous type 1 Brugada pattern and/or cardiac syncope.

As seen in our patient's ECG (Brugada type 3 pattern), the appearance of "saddle-back morphology" aspect in leads V1 and V2 mimicking ST elevation myocardial infarction is an additional diagnostic and therapeutic challenge in COVID-19 patients presenting with chest pain, as recently reported [6]. We excluded acute myocardial infarction due to incompatible electrocardiographic criteria and absence of wall motion abnormalities on echocardiography. Recently, a multicentric, crosssectional, retrospective study described the electrocardiographic features of 431 hospitalized critically ill COVID-19 patients [13]. Thirty percent of the patients had ECG signs suggesting acute right ventricular pressure overload. In line with this finding, considering the lung parenchymal destruction of our patient seen in chest tomography, we think that the electrocardiographic finding is due to the pulmonary hypertension detected on the echocardiogram. Therefore, even if he had survived, in our opinion an implantable cardioverter defibrillator wouldn't be indicated at least without provocative testing. According to our experience, in addition to assessing the severity of lung parenchymal involvement, threshold to run an ECG should be low in febrile patients with suspected COVID-19. Also, all patients with high fever and syncope especially those with known or suspected Brugada ECG patterns may warrant more aggressive antipyretic therapy and serial screening ECGs.

\section{Conclusion}

We present this case to increase awareness of the potential association between COVID-19 and the development of ST elevation pattern on ECG. Since many different pathophysiological mechanisms of Covid disease have been proposed, it is important to look at it from a broad physio pathological perspective while evaluating the clinical manifestations related to Covid 19 disease.

\section{Acknowledgement}

We Thank Dr Hasan Arı for its advices and suggestions on the manuscript. 


\section{Conflict of Interest}

No conflict of interest.

\section{References}

1. Rajkumar RP (2020) COVID-19 and mental health: A review of the existing literature. Asian J Psychiatr 52: 102066.

2. Keller DI, Rougier JS, Kucera JP, Benammar N, Fressart V, et al. (2005) Brugada syndrome and fever: genetic and molecular characterization of patients carrying SCN5A mutations. Cardiovasc Res 67: 510-519.

3. Lee CCE, Ali K, Connell D, Mordi IR, George J, et al. (2021) COVID-19Associated Cardiovascular Complications.

4. Angeli F, Spanevello A, De Ponti R, Visca D, Marazzato J, et al (2020) Electrocardiographic features of patients with COVID-19 pneumonia. Eur J Intern Med 78: 101-106.

5. Polito MV, Silverio A, Bellino M, Iuliano G, Di Maio M, et al (2021) Cardiovascular Involvement in COVID-19: What Sequelae Should We Expect. Cardiol Ther 30: 1-20.

6. Pasquetto G, Conti GB, Susana A, Leone LA, Bertaglia E (2020) Syncope, Brugada syndrome, and COVID-19 lung disease. J Arrhythm 36: 768-770.

7. Liu PP, Blet A, Smyth D, Li H (2020) The Science Underlying COVID-19: Implications for the Cardiovascular System. Circulation 142: 68-78.
8. Zuo Y, Yalavarthi S, Shi H, Gockman K, Zuo M, et al. (2020) Neutrophil extracellular traps (NETs) as markers of disease severity in COVID-19. JCI Insight 5(11): e138999.

9. Wu CI, Postema PG, Arbelo E, Behr ER, Bezzina CR, et al. (2020) SARSCoV-2, COVID-19, and inherited arrhythmia syndromes. Heart Rhythm 17: $1456-1462$.

10. Brugada J, Campuzano O, Arbelo E, Sarquella-Brugada G,Brugada R (2018) Present Status of Brugada Syndrome: JACC State-of-the-Art Review. J Am Coll Cardiol 72: 1046-1059.

11. Dumaine R, Towbin JA, Brugada P, Vatta M, Nesterenko DV, et al. (1999) Ionic mechanisms responsible for the electrocardiographic phenotype of the Brugada syndrome are temperature dependent. Circ Res 85: 803809.

12. Amin AS, Meregalli PG, Bardai A, Wilde AA, Tan HL (2008) Fever increases the risk for cardiac arrest in the Brugada syndrome. Ann Intern Med 149: 216-218.

13. Bertini M, Ferrari R, Guardigli G, Malagu M, Vitali F, et al. (2020) Electrocardiographic features of 431 consecutive, critically ill COVID-19 patients: an insight into the mechanisms of cardiac involvement. Europace 22: 1848-1854. 